\title{
Average Surface Roughness Measurement Using Photometric Stereo Method
}

\author{
Thammarat Somthong ${ }^{*}$, Qing-Ping Yang \\ College of Engineering, Design and Physical Sciences, Brunel University London, UK
}

Copyright $(2016$ by authors, all rights reserved. Authors agree that this article remains permanently open access under the terms of the Creative Commons Attribution License 4.0 International License

\begin{abstract}
This paper presents a technique for measuring surface roughness $(R a)$, using photometric stereo method. The method utilizes three or more images of the measured surface from the same view point, taken sequentially under different illumination conditions. The scenes captured by the camera were reconstructed by the least square method to obtain surface normal from surface topography. Three-dimensional surface textural patterns were recovered from those surfaces normal. The system was validated with three standard surface roughness specimens. The $R a$ calculated from the recovered surface was compared with the nominal value of the standard surface roughness. The best coefficient of the slant angles can also be determined through the linear regression. The experimental results indicate that the technique can be applied to well recover standard surface roughness.
\end{abstract}

Keywords Surface Texture, Photometric Stereo, Measurement, Imaging Techniques

\section{Introduction}

Computer graphics techniques have been extensively popular in the measurement system. The quality of components produced is of major concern in industrial manufacturing, and it is closely related to dimensional accuracy, form and surface finish. Surface topography, especially surface roughness, has an essential role to determine the functional performance of machine parts. The measurement of engineering surface roughness is becoming increasingly important. The traditional way of measuring surface topography is mechanical - most existing roughness standards are defined using stylus instruments that normally use a diamond stylus. Stylus can usually scratch the measured surface and may not be suitable when the measured surfaces are very soft, for example, in biological systems or polymers. In the last few decades, alternative methods rather than stylus have been developed. Those can be loosely divided into optical methods (such as vertical scanning interferometry) and non-optical methods (such as scanning tunneling microscopy) [1] [3].

Recently, photometric stereo method has been considered as a technique for roughness measurement. The photometric stereo is a method for evaluation of shape and reflectance of an object using three or more images under different lighting positions [7]. The method uses different lighting conditions to mainly measure the gradient field of the surface, which is calculated from an array of surface normal. The gradient field can be therefore considered to be the measurement results. The surface texture can be calculated from the obtained depth information [2] [4] [5].

In this paper, we consider the slant and tilt angles which are suitable for measuring different roughness ranging from $12.5 \mu \mathrm{m}$ to $50 \mu \mathrm{m}$. The $R a$ is then reconstructed by combining three images, each at a tilt angle with the object. By using the reconstructed $R \mathrm{a}$ of the objects, parameters of a reflection model are estimated in a robust manner. The key point of the proposed method is that the linear regression technique was applied to find the best coefficient between image intensities and roughness.

\section{Operating Principle}

\section{The Reflectance Model}

Intensity values are known from image sets that recorded a reflected radiance from the illuminated objects. There are many contributing factors relating to the reflected radiance when the incident light has constant direction and intensity, e.g. the physical properties and shape of the measured object. If the surfaces are rough enough so that all incident light beams are diffusively reflected to all directions, these surfaces are so-called lambertian surfaces [6]. According to the lambertain reflectance model, the light $(I)$ reflected from an object's surface is related to the surface albedo $(\rho)$ and the cosine angle of the incident light $(k)$ as given in Eq. (1).

$$
I=\rho k \cos \left(\theta_{i}\right)
$$

\section{Photometric Stereo (PS)}

The photometric stereo method was firstly proposed by Woodham in 1980. It is a method that calculates local surface reflection and orientation through the locational of incident light source, with several images taken from a constant viewing direction [2] [7]. The basic idea of the PS method is to solve Eq. (2) for the unknown surface normal. 


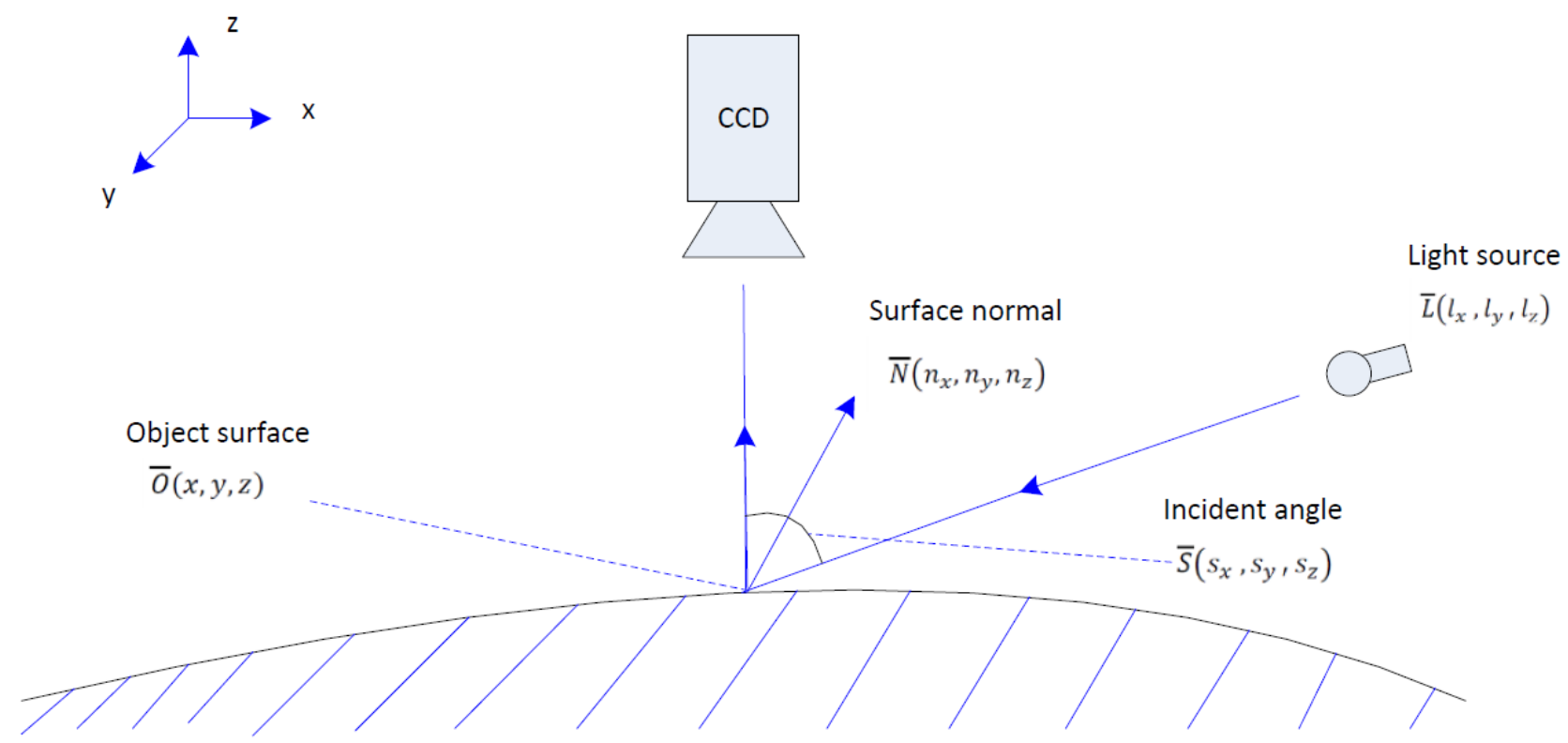

Figure 1. Photometric stereo system

When three images with the same view are taken under different lighting directions, three reflectance maps are provided for solving a linear system of three unknown surface normals $\left(n_{x}, n_{y}, n_{z}\right)$. The cosine of the incident angle can also be denoted as dot product, as shown in Eq. (2), where $\vec{S}$ represents the unit vector of light source direction and the unit vector $\vec{N}$ represents the surface normal:

$$
I=\rho(\vec{S} \cdot \vec{N})
$$

In case of three images being utilized by the measurement system,

$$
\left[\begin{array}{l}
I_{1} \\
I_{2} \\
I_{3}
\end{array}\right]=\rho\left[\begin{array}{lll}
s_{x 1} & s_{y 1} & s_{1 z} \\
s_{x 2} & s_{y 2} & s_{2 z} \\
s_{x 3} & s_{y 3} & s_{3 z}
\end{array}\right]\left[\begin{array}{l}
n_{x} \\
n_{y} \\
n_{z}
\end{array}\right]
$$

In Eq. (3), $\left(I_{1}, I_{2}, I_{3}\right)$ represent the image intensity values at image location $(x, y)$. Unknown surface albedo is denoted by $\rho$. The unknown components of the surface normal is represented by $\left(n_{x}, n_{y}, n_{z}\right)$, and $\left(s_{x}, s_{y}, s_{z}\right)$ are the known components of light source vector, where numbers 1, 2 and 3 indicate light direction. To solve the unknown surface reflectance factor and surface normal, the following relation has to be used:

$$
\vec{I}(x, y)=\rho(x, y)[S] \vec{N}(x, y)
$$

To determine the surface normal, $\mathrm{N}$, in Eq. (4), the following relation can be utilized from Eq. (5), where the lighting directions, $\mathrm{S}$, must not be coplanar to a plane.

$$
\vec{N}(x, y)=\frac{1}{\rho(x, y)}[S]^{-1} \vec{I}(x, y)
$$

The least squares technique can be utilized to calculate Pseudo-Inverse and local surface gradients $p(x, y)$ and $q(x, y)$. The local surface normal $\vec{N}(x, y)$ can also be calculated from the Pseudo-Inverse using Eqs. (6)-(8) where $\vec{M}(x, y)=\left(m_{1}(x, y), m_{2}(x, y), m_{3}(x, y)\right)$.

$$
\begin{gathered}
\vec{M}(x, y)=\rho(x, y) \vec{N}(x, y)=\left([S]^{T}[S]\right)^{-1}[S]^{T} \vec{I}(x, y) \\
p(x, y)=\frac{m_{1}(x, y)}{m_{3}(x, y)}, q(x, y)=\frac{m_{2}(x, y)}{m_{3}(x, y)} \\
N(x, y)=\frac{p(x, y), q(x, y), 1}{\sqrt{p(x, y)^{2}+q(x, y)^{2}+1}}
\end{gathered}
$$

\section{Surface Texture}

Surface measurement usually yields the information in two types, i.e. the roughness and the waviness. These parameters can be used to explain and predict the performance and problems of machine tools. Filtering techniques are applied to separate roughness and waviness from the surface topography.

The surface profile parameters are defined by ISO 4287:1997 which encompasses five groups of texture parameters, including amplitude parameters (peak and valley), amplitude parameter (average of ordinates), spacing parameters, hybrid parameters, and curves and related parameters [11].

The surface roughness parameter used throughout this study is the arithmetical mean of the surface profile $(R a)$. This parameter is also the most widely used surface finish parameter by researchers and industries. $R a$ is the arithmetic average of the absolute value of the heights of roughness irregularities from the mean value measured [8] [9, p.7]. It can be described in mathematical function as

$$
R_{a}=\frac{1}{N} \sum_{i=1}^{N}\left|Z_{i}\right|
$$

where $Z_{i}$ is the height of roughness irregularities from the mean value and $N$ is the number of measured points in a sampling length. $R a$ value can be calculated from the mean height [9]. 


\section{System Designs}

\section{Optimal Lighting Position}

Spence and Chantler [6] worked with three lights of equal slant and using numerical optimisation they concluded that the normals are best recreated when the light sources are $120^{\circ}$ apart, and of slant $55^{\circ}$. This corresponds to orthogonal light directions and is in full agreement with the results derived from the theory presented here.

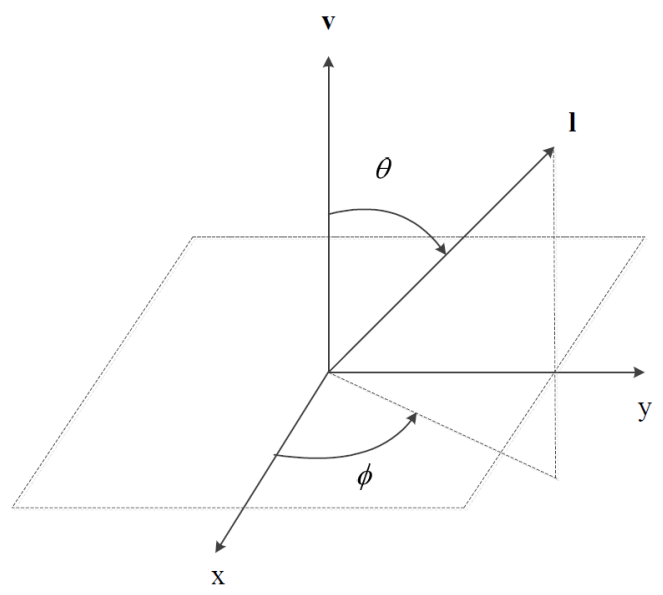

Figure 2. Slant and tilt of a light source
In figure 2, the angle between light source (I) and viewing vector $(\mathbf{v})$ is the slant angle $(\theta)$. Tilt $(\phi)$ is the angle between the projection of $\mathbf{I}$ onto the viewing plane $(x-y)$ and the $\mathrm{x}$-axis.

\section{Proposed Photometric Stereo System}

Fig. 3 illustrates the PS system set up on the Coordinate measuring machine (CMM) for surface roughness measurement. The roughness standard is mounted at centre of the measuring table. The light source is assumed to be a point source with a constant incident illumination over the scene.

The purpose of the experimental work was to investigate the practical application of PS techniques for measuring $R a$ from surface texture. The nominal values of standard surface roughness which included $12.5 \mathrm{um}, 25$ and 50 um were applied to validate the PS system. Three differing standard surface roughness was viewed by a vision system constrained pose as their position. The light source was attached with the CMM's probe, which was able to move tilt and slant angles. The sensitivity of the technique to the slant angle was investigated. Three slant angles were examined: $\theta$ $=37.5^{\circ}, 45^{\circ}$ and $52.5^{\circ}$. The optimal illumination configuration with regard to tilt angle is considered to be an intuitive result since the three vectors are equally spaced at $120^{\circ}$ apart.

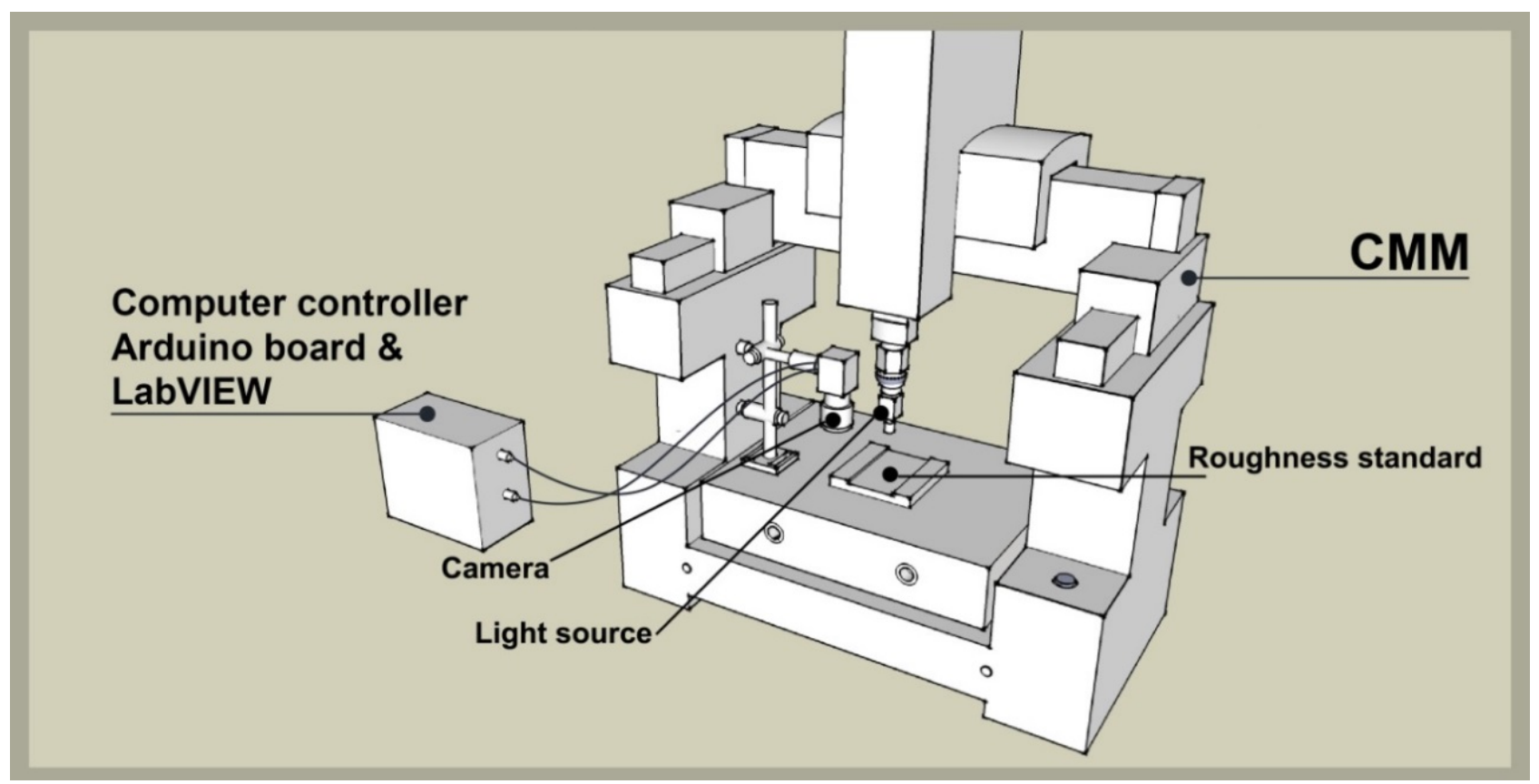

Figure 3. The PS system on the CMM for measuring surface texture 


\section{Measurement Method}

Using the PS method for measuring surface texture can be written as block diagrams in Fig. 4.

The block diagrams show the six steps for measurement method. The Kingbright $52 \mathrm{~mm}$ cluster is used as the light source to create a stable and well-controlled illumination condition. The images with a dimensional size $512 \times 320$ pixel are captured from the Basler Ace Digital Gigabit Ethernet camera with a $16 \times$ optical zoom lens. Those images acquired from each tilt angle are precisely performed by the CMM's movement. In case of mark images, the area of interest is selected by Matlab programming. Gradient space plots are generated and analysed for all standard surface roughness. The surface normal is therefore calculated by the least squares technique following by Eq. (8). Therefore, accurate shape reconstruction results can be achieved. Twelve lines on the scene coming from pixel points are then chosen for intensity calculation and compared with the roughness standards.

\section{Experimental Results}

\section{Estimated Average Surface Roughness Profile}

In this research, all images were acquired in a darkroom so that the influence of unwanted extra light was prevented in the scenes. The three nominal values of surface roughness standard Type D following ISO 5436-1 were utilized as the reference of our system.

The original scenes of the surface roughness standard fewer than three light directions are shown in Fig. 6. The idealised simulated surface roughness standard from the PS system is shown in Fig. 7. The 3D recovery of the surface roughness standard measured using from the PS system is illustrated in Fig. 8.

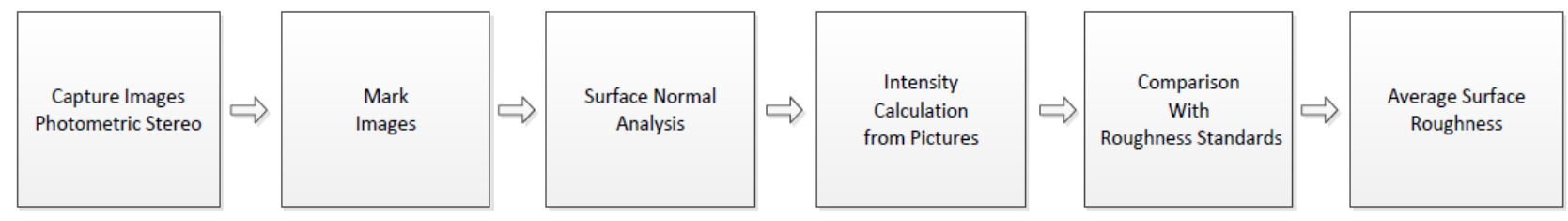

Figure 4. Inspection strategy

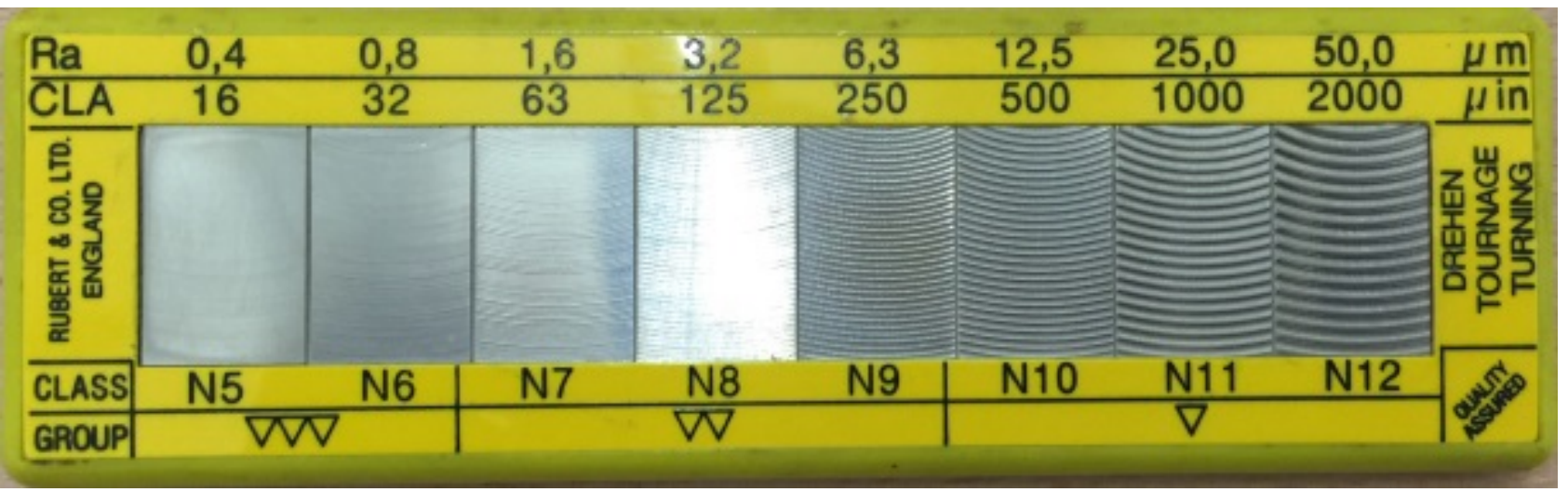

Figure 5. Surface roughness standard Type D following ISO 5436-1

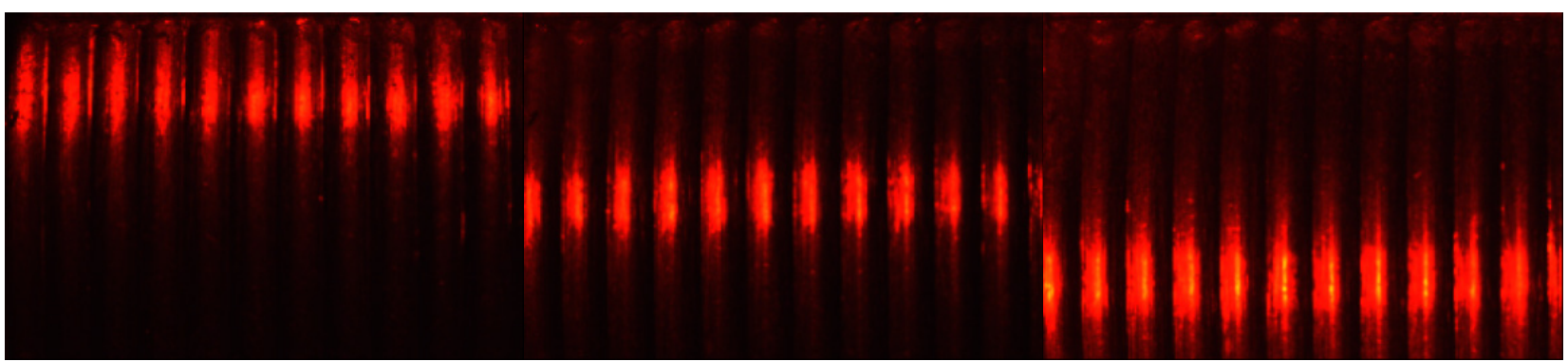

Figure 6. Three images of surface roughness standard illuminated from tilt angle $0^{\circ}, 120^{\circ}$ and $240^{\circ}$ 


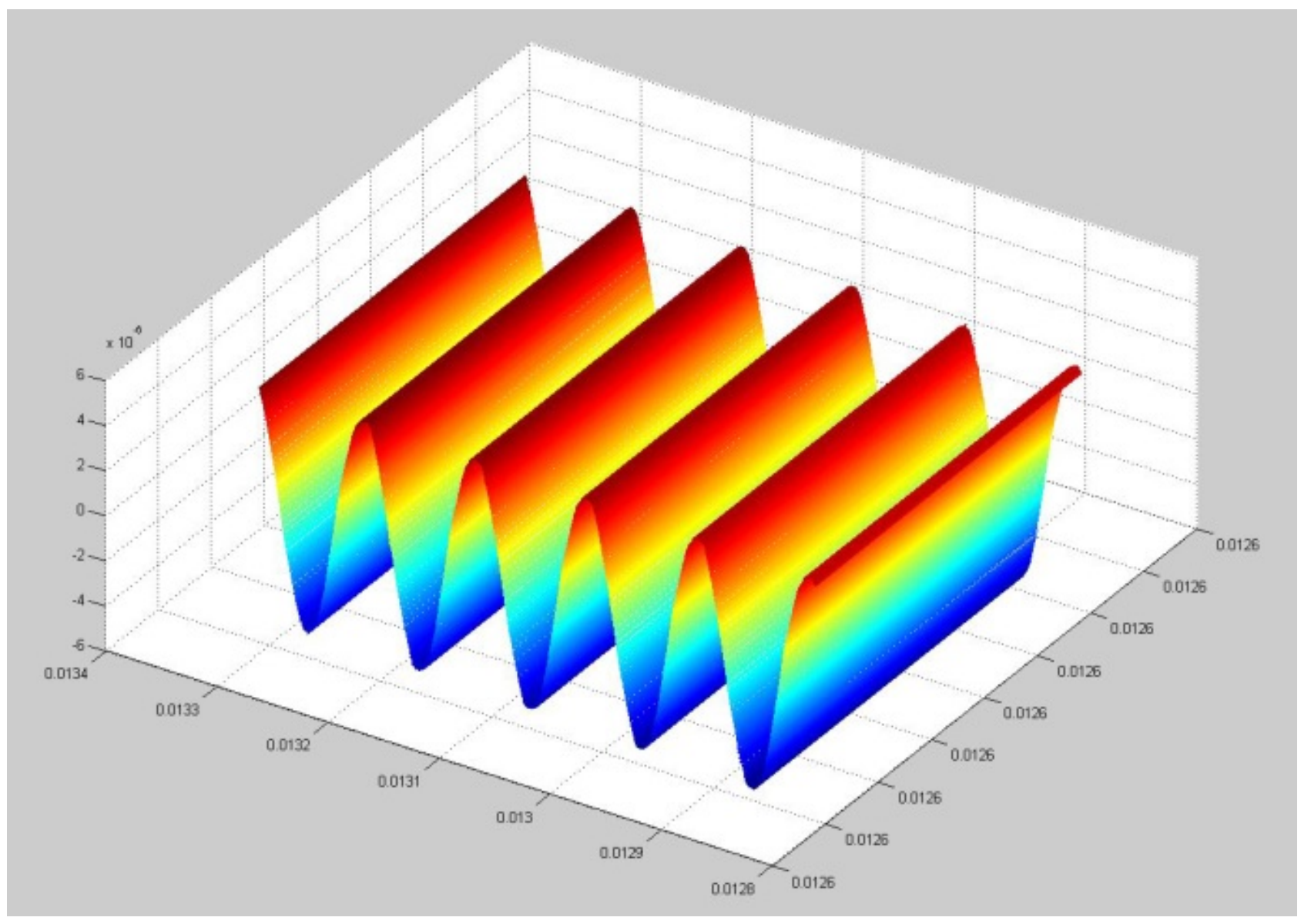

Figure 7. Idealised simulated surface roughness standard

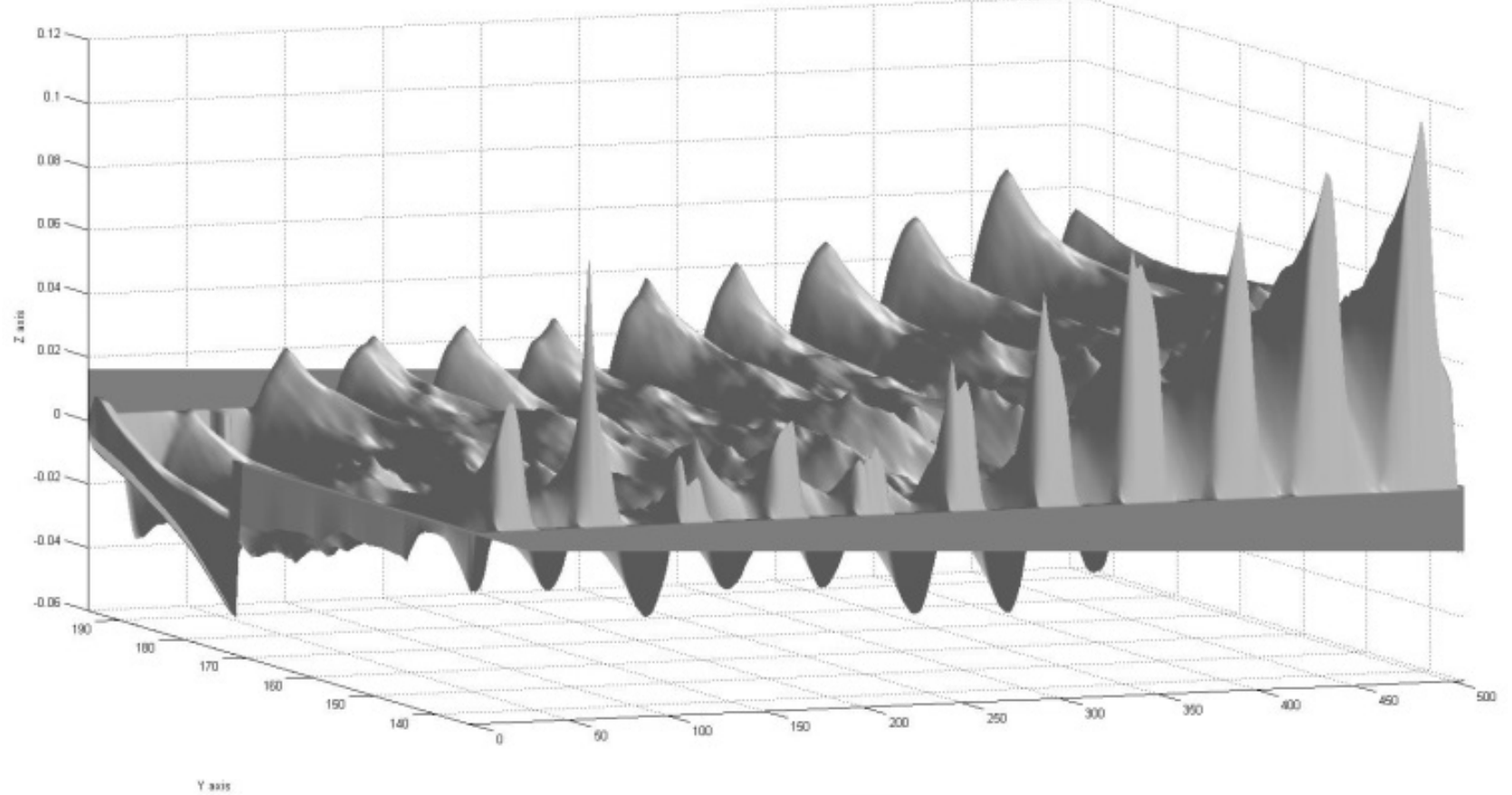

Figure 8. Recovered three dimensional surface roughness standard 

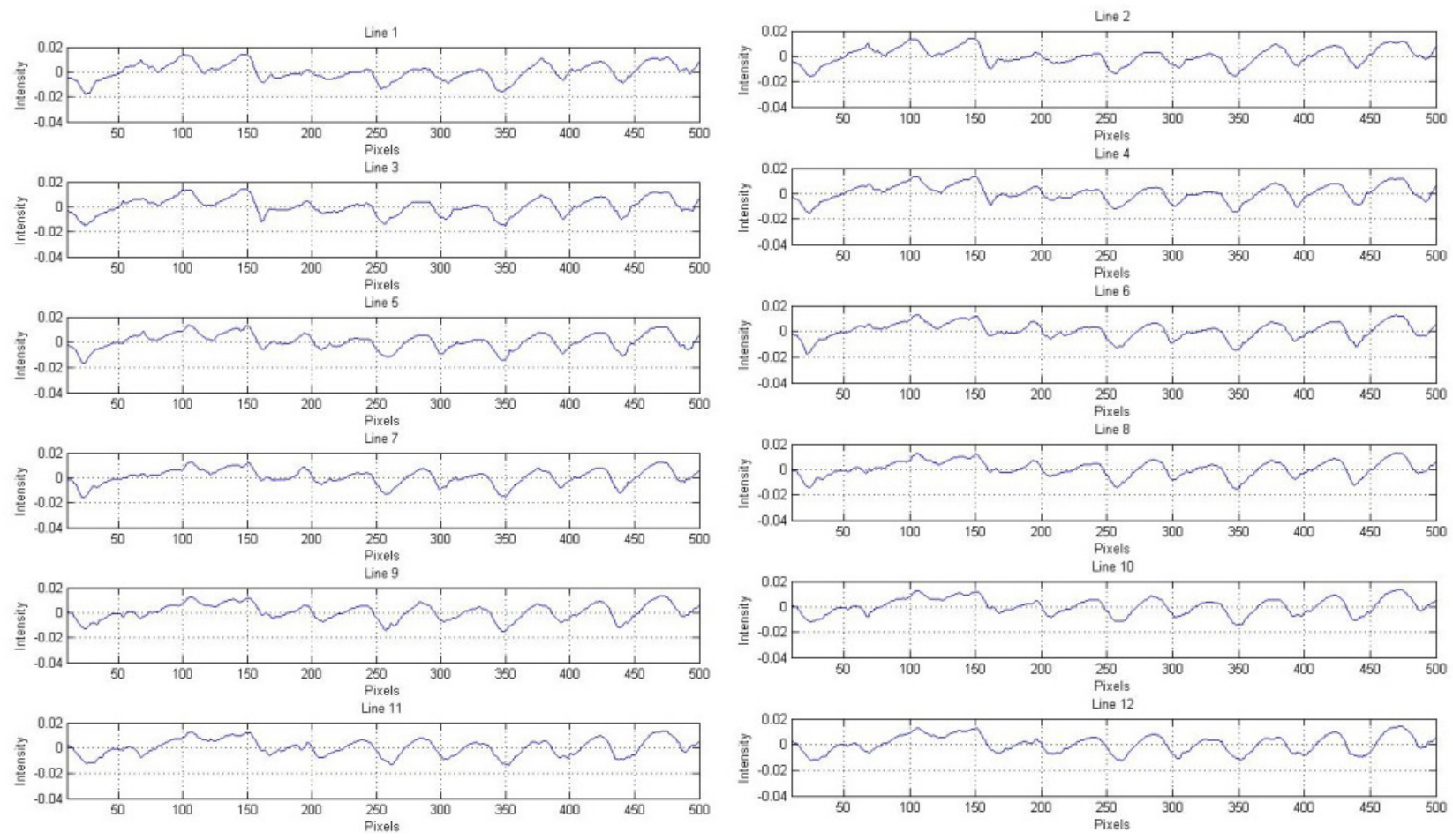

Figure 9. Surface roughness profiles

\section{Computing Average Surface Roughness Using Photometric Stereo Method}

The sample profiles are captured, with its centre coincides with the image centre. Each image acquired from the camera covers an area of specimen around $512 \times 340 \mathrm{~mm}$. The average value 12 lines from the simple profiles are then calculated according to ISO 4288:1996.For each value of surface texture, the best coefficient of linear regression is verified with the profiles. The results of measurement are summarized in Table 1 as the coefficients of determination between the estimated $R a$ by the PS system against the nominal value from surface roughness standard.

Table 1. Linear Regression between nominal value of surface roughness standard and PS technique results under different slant angles

\begin{tabular}{ccc}
\hline $\begin{array}{c}\text { Slant angle } \\
(\theta)\end{array}$ & Linear regression model & $\begin{array}{c}\text { Coefficient of } \\
\text { determination }\end{array}$ \\
\hline $37.5^{\circ}$ & $\mathrm{Ra}_{\text {std }}=-3040.5 \mathrm{Ra}_{\mathrm{PS}}+438.42$ & 0.94 \\
\hline $45^{\circ}$ & $\mathrm{Ra}_{\text {std }}=-1632.4 \mathrm{Ra}_{\mathrm{PS}}+243.23$ & 0.97 \\
\hline $52.5^{\circ}$ & $\mathrm{Ra}_{\text {std }}=-1116.3 \mathrm{Ra}_{\mathrm{PS}}+114.71$ & 0.99 \\
\hline
\end{tabular}

Where $\mathrm{Ra}_{\mathrm{PS}}$ is roughness standard profiles which are averaged from 12 lines from image measurement system, and $\mathrm{Ra}_{\text {std }}$ is nominal values which are identified surface roughness standard. Three values of surface roughness standards were utilized for evaluation of the PS system. The best coefficient was found at $52.5^{\circ}$ of slant angle. This equation was offered for roughness average estimation form recovered surface. Furthermore, more information on surface characteristics would be presented from three-dimensional (3D) surface textural patterns of the surface roughness standard.

\section{Conclusions}

The experiment results have shown that the new approach for textural examination using photometric stereo can measure surface roughness of work pieces. However, the results from comparison experiments would be able to increase accuracy by surface roughness standard calibration. In fact, more experimental cases have to be considered and compared with the present method of analysis. In addition, the measurement uncertainty using the PS system should be calculated.

\section{REFERENCES}

[1] S. Damodarasamy and S. Raman, Texture analysis with computer vision. Computer in Industry 16 (1991), pp. 25-34.

[2] M.L. SMITH, The analysis of surface texture using photometric stereo acquisition and gradient space domain mapping. Image and Vision Computing 17 (1999), pp. 1009-1019.

[3] G. A. Al-Kindi, R. M. Baul, K. F Gill, An application of machine vision in the automated inspection of engineering surfaces. International Journal of Production Research (1992), pp. 241-253.

[4] M.L. Smith, G. Smith, T. Hill, Gradient space analysis of surface defects using a photometric stereo derived bump map. Image and Vision Computing 17 (1999), pp. 321-332.

[5] E. North Coleman, JR., R. JAIN, Obtaining 3-dimensional shape of textured and specular surfaces using four-source photometry. Computer graphics and image processing 18 
(1982), pp. 309-328.

[6] A. Spence and M. Chantler, Optimal illumination for three-image photometric stereo acquisition of texture, Proceedings of the 3rd International Workshop on Texture Analysis and Synthesis (2003), 89-94.

[7] R.J. Woodham, Determining Surface Curvature with Photometric Stereo, IEEE International Conference on Robotics and Automation (1989), pp. 36 - 42.

[8] D. Whitehouse, Surface and Their Measurement, Hermes Penton, London, 2002.

[9] R. Leach, Good Practice Guide No.37 The Measurement of Surface Texture using Stylus Instruments, National Physical
Laboratory, United Kingdom, 2001

[10] ISO10360-1. Geometrical Product Specification (SPC). Acceptance and reverification tests for coordinate measuring machines (CMM). Part 1: Vocabulary. International Organization for Standardization; 2000.

[11] ISO 4287:1997 Geometrical Product Specifications (GPS) Surface texture: Profile method -Terms, definitions and surface texture parameters

[12] ISO 4288:1996 Geometrical Product Specifications (GPS) Surface texture: Profile method-Rules and procedures for the assessment of surface texture

[13] ISO 5436:2000 Geometrical Product Specifications (GPS) Surface texture: Profile method; Part1: Material measures 